\title{
The Expanding Digital Divide: Digital Health Access Inequities during the COVID-19 Pandemic in New York City
}

\author{
Chukwuma N. Eruchalu • Margaret S. Pichardo - Maheetha Bharadwaj • Carmen B. \\ Rodriguez • Jorge A. Rodriguez • Regan W. Bergmark • David W. Bates • \\ Gezzer Ortega $(\mathbb{D})$
}

Accepted: 15 December 2020 /Published online: 20 January 2021

(C) The New York Academy of Medicine 2021

\section{Introduction}

As telemedicine use for healthcare delivery during the COVID-19 pandemic has grown, access to technology has emerged as a fundamental determinant of access to care. Accordingly, digital access is now being recognized as a social determinant of health [1]. Over 21 million individuals in the USA lack broadband internet access, limiting access to telemedicine [2, 3]. New York State (NYS) has been one of the early epicenters of the pandemic in the USA. While NYS has a $99.9 \%$ broad-

C. N. Eruchalu • M. Bharadwaj

Harvard Medical School, Boston, MA, USA

M. S. Pichardo

Howard University College of Medicine, Washington, DC, USA

\section{S. Pichardo}

Department of Chronic Disease Epidemiology, Yale School of Public Health, New Haven, CT, USA

\section{B. Rodriguez}

Department of Epidemiology, Columbia University Mailman

School of Public Health, New York, NY, USA

\section{J. A. Rodriguez $\cdot$ D. W. Bates}

Division of General Internal Medicine, Department of Medicine, Brigham and Women's Hospital, Harvard Medical School,

Boston, MA, USA

R. W. Bergmark • G. Ortega $(\bowtie)$

Center for Surgery and Public Health, Department of Surgery,

Brigham and Women's Hospital, Harvard Medical School,

Boston, MA, USA

e-mail: Gortega1 @bwh.harvard.edu band internet infrastructure, nearly one-third of New York City (NYC) households, or approximately 2.2 million residents, lack a home broadband internet subscription [4-6]. Furthermore, significant disparities in broadband internet access exist for NYC residents who are low income, racial/ethnic minority, over the age of 65 , or have a non-English primary language. Digital literacy is also lower among these populations [5-7].

In New York City, neighborhood-level disparities in COVID-19 infectivity and mortality have largely been attributed to race/ethnicity and socioeconomic differences [8]. However, the influence of digital access inequities on these COVID-19 disparities has not been examined quantitatively. For racial/ethnic minority, low-income, and other vulnerable populations that are disproportionately burdened by the digital divide, disparities in digital access may compound other forms of structural disadvantage, exacerbating COVID-19 and health access disparities [6]. In the following commentary, we explore policy considerations and solutions to reduce the impact of the digital divide on vulnerable populations during the COVID-19 pandemic and beyond.

\section{Increasing Access to Broadband}

NYC neighborhoods with poor access to broadband internet tend to also have higher proportions of racial/ ethnic minorities, higher poverty rates, and lower educational attainment, sociodemographic factors that have been linked to excess COVID-related hospitalizations 
and mortality $[8,9]$. To prevent the exacerbation of preexisting disparities, the rapid expansion of telemedicine must be paralleled by a commensurate expansion of access to broadband internet. In response to the ruralurban digital divide, the Federal Communications Commission (FCC) launched the Digital Opportunities Data Collection mapping project to identify broadband coverage gaps in rural areas and established the Rural Digital Opportunity Fund, which allocated $\$ 20.4$ billion to expand broadband infrastructure to rural areas [10]. Additionally, through the Connect America Fund, the FCC partnered with New York State to provide a combined $\$ 390$ million to support rural broadband expansion [10]. Similar broadband mapping initiatives and federal-state financial partnerships should be enacted to identify low-coverage regions within urban areas and increase broadband access within those communities.

Government subsidies for broadband subscribership and data charge subsidies for mobile health applications will help reduce cost barriers to individual patients [1]. Broadband access can be further expanded by leveraging existing community infrastructure and social programs. For example, broadband hotspots should be established in public spaces, such as libraries, schools, and community centers [11]. Efforts should be made to relax or waive re-enrollment requirements for initiatives such as the Lifeline Program, which provides telephone and internet subsidies to low-income individuals and people enrolled in certain federal assistance programs, in order to prevent subscribers from losing access due to COVID-related disruptions [12]. Finally, health providers and health systems can help accelerate broadband deployment in low-resource communities by supporting policies that reduce financial, bureaucratic, and structural barriers to the expansion of required infrastructure, such as digital redlining of less economically profitable neighborhoods $[2,10,13]$.

\section{Increasing Access to Technology}

During the COVID-19 pandemic, virtual access to providers has become vital for respiratory symptom screening and triage. Thus, in addition to greater access to broadband internet, vulnerable communities will require increased access to technological devices to engage with telehealth platforms. Although significant increases in ownership of both smartphones and computing devices have been observed in the past decade, disparities persist across race/ethnicity, income, education, and age groups [14]. During the pandemic, the NYC Department of Education has provided over 320,000 tablets to students in attempts to narrow the digital divide in education [15]. Hospitals should support similar device distribution programs for patients at risk of becoming disconnected from virtual care. Device ownership screening should occur as part of routine patient intake, similar to electronic health record-based social determinants of health screening used to identify other resource gaps [16]. Screening questions should further assess the number of devices available in the household, the types of devices, and whether those devices have sufficient bandwidth to support video conferencing for telehealth visits.

\section{Improving Digital Literacy}

Access to accurate, culturally sensitive information about COVID-19, much of which is disseminated online, and the ability to adhere to evidence-based safety guidelines are fundamental to limiting viral spread during the pandemic. Populations with low digital literacy are less capable of effectively utilizing technological devices or accessing the internet to obtain health information, making them less able to remain updated on official safety recommendations concerning COVID-19 $[11,17]$. Furthermore, racial/ethnic minorities, older adults, and individuals with lower educational attainment are less likely to engage with online patient portals to participate in chronic disease management, even with adequate internet and computer access [18]. Thus, digital and health literacy likely contribute to disparities in the use of mobile health applications even with sufficient access to technology.

Hospitals should partner with community colleges, public libraries, and other community-based organizations to develop education and skills-building programs to address digital and health literacy gaps [11]. These training programs should be designed according to needs identified by community stakeholders, available in multiple languages, and implemented in a variety of forms, such as in-person or virtual classes, real-time online help, and home assistance from trained digital literacy workers [11]. Digital literacy training will allow vulnerable patient groups to better access and interpret online health information. 


\section{Implementing Equitable Telemedicine Platforms}

Although telemedicine provides advantages for medically underserved communities, such as limiting risk of disease transmission, decreasing time invested in obtaining healthcare, reducing transportation barriers to attending in-person appointments, and potentially expanding access to specialty care, it is not a panacea for healthcare access inequities [19]. Following the widespread implementation of telemedicine during the pandemic, primary care visits by patients with limited digital access, including racial/ethnic minority groups, patients over age 65 , and patients with non-English language preference, have disproportionately decreased, raising concern of potential delayed management of chronic diseases as well as delayed COVID-19 diagnoses [20]. Disruptions in chronic disease screening, prevention, and treatment will most significantly impact populations with limited access to telemedicine [21]. Although increasing digital access and digital literacy will help vulnerable patients obtain virtual visits, language, cultural, and logistical barriers may impair the quality of care $[19,22]$.

Additionally, logistical and privacy concerns emerge when patients attend appointments from home. Access to quiet, private spaces for discussion of sensitive health information can be limited for patients living in crowded, multi-generational households. Furthermore, virtually inviting physicians into their home environment may be viewed by patients as an invasion of privacy [19]. Some patients have also raised concerns about the security of personal health information that is shared through virtual platforms [19]. Health systems must incorporate flexibility in modalities for virtual visits by allowing patients to turn their video off or conducting audio-only visits by telephone when clinically appropriate. Healthcare providers can partner with local organizations such as public libraries and community centers to supply private office spaces from which patients can attend telehealth visits. Finally, digital literacy workers can help patients better understand the privacy policies and security infrastructure of mobile health services.

Furthermore, health systems should develop culturally and linguistically inclusive digital health platforms and track their usage among the sociodemographic groups most affected by the digital divide [23]. These populations should be included in the development of mobile health tools. Health providers can advocate for federal mandates like the Culturally and Linguistically Appropriate Service Standards to formalize guidance on promoting digital health equity, such as by expanding the availability of interpreter services for telehealth visits [23]. Additionally, conference calls that incorporate family members or caregivers with higher digital literacy in the medical visit can help vulnerable patients navigate technical barriers to engaging with virtual platforms, promote effective communication between patients and providers, and equip patients with additional home support for chronic disease management during a challenging time.

Finally, as we address virtual health disparities, we must recognize the clinical limitations of telemedicine. Although virtual visits can be effective in specialties that primarily utilize verbal communication and visual physical examination, remote patient assessment may not be clinically appropriate in other scenarios, such as surgical consultations [19]. Even as telemedicine expands and mobile health technology continues to advance, there will remain a role for in-person visits for certain clinical problems.

\section{Conclusion}

The COVID-19 pandemic has highlighted that digital access is now a social determinant of health and a prerequisite for access to both COVID-related and non-COVID care $[1,19]$. Accordingly, individual providers and health systems must combat digital health inequities by collaborating with community-based, state, and federal organizations to increase access to broadband internet and computing devices, improve digital literacy, and mitigate disparities in telehealth. In order to fully address the disparate impact of the pandemic and promote equity in the era of telemedicine, we must bridge the digital divide.

Acknowledgements None.

Funding None.

\section{References}

1. Fridsma DB. AMIA response to FCC notice on accelerating broadband health tech availability. Published online May 24, 2017. https://www.amia.org/sites/default/files/AMIA- 
Response-to-FCC-Notice-on-Accelerating-BroadbandHealth-Tech-Availability.pdf. Accessed 26 July 2020.

2. 2019 Broadband Deployment Report. Federal Communications Commission; 2019:331. https://docs.fcc. gov/public/attachments/FCC-19-44A1.pdf. Accessed 2 July 2020 .

3. Wilcock AD, Rose S, Busch AB, et al. Association between broadband internet availability and telemedicine use. JAMA Intern Med. 2019;179(11):1580-2. https://doi.org/10.1001 /jamainternmed.2019.2234.

4. Governor Cuomo Announces Round III of Nation-Leading New NY Broadband Program to Bring High-Speed Internet Access to All New Yorkers. Governor Andrew M. Cuomo. Published January 31, 2018. https:/www.governor.ny. gov/news/governor-cuomo-announces-round-iii-nationleading-new-ny-broadband-program-bring-high-speed. Accessed 2 July 2020.

5. Gamino, Jr. M. Truth in broadband: access and connectivity in New York City. NYC Mayor's Office of the Chief Technology Officer; 2018:66. https://tech.cityofnewyork. us/wp-content/uploads/2018/04/NYC-ConnectedBroadband-Report-2018.pdf. Accessed 22 Jul 2020.

6. Stringer SM. Census and the city: overcoming NYC's digital divide in the 2020 census. New York City Comptroller: Bureau of Policy \& Research; 2019:24. https://comptroller. nyc.gov/wp-content/uploads/documents/Census_and_The City_Overcoming_NYC_Digital_Divide_Cénsus.pdf. Accessed 22 Jul 2020.

7. Mamedova S, Pawlowski E. A description of U.S. adults who are not digitally literate. U.S. Department of Education; 2018:33. https://nces.ed.gov/pubs2018/2018161.pdf. Accessed 24 June 2020.

8. COVID-19: data summary. NYC Health. https://www1.nyc. gov/site/doh/covid/covid-19-data.page\#download. Accessed 25 May 2020

9. Wadhera RK, Wadhera P, Gaba P, et al. Variation in COVID-19 hospitalizations and deaths across New York City boroughs. JAMA. Published online April 29, 2020. https://doi.org/10.1001/jama.2020.7197.

10. Bridging the digital divide for all Americans. Federal Communications Commission. Published July 27, 2017. https://www.fcc.gov/about-fcc/fcc-initiatives/bridgingdigital-divide-all-americans. Accessed 16 Sept 2020.

11. Institute of Museum and Library Services. Building digital communities: getting started. Institute of Museum and Library Services; 2012:36. https://www.imls.gov/assets/1 /AssetManager/BuildingDigitalCommunities.pdf. Accessed 26 July 2020.

12. Lifeline support for affordable communications. Federal Communications Commission. Published March 4, 2016. https://www.fcc.gov/lifeline-consumers. Accessed 16 Sept 2020.

13. Penarroyo C, Lindquist S, Miller R. Mapping Detroit's digital divide. Urban Collaboratory: University of Michigan; 2018:37. https://www.urbanlab.umich. edu/project/mapping-detroits-digital-divide/. Accessed 9 Oct 2020.

14. Demographics of mobile device ownership and adoption in the United States. Pew Research Center: Internet \& Technology. Published June 12, 2019. https://www. pewresearch.org/internet/fact-sheet/mobile/. Accessed 2 July 2020.

15. Return to school 2020: iPad requests. NYC Department of Education. https://www.schools.nyc.gov/learning/learn-athome/technical-tools-and-support/ipad-requests. Accessed 16 Sept 2020.

16. Buitron de la Vega P, Losi S, Sprague Martinez L, et al. Implementing an EHR-based screening and referral system to address social determinants of health in primary care. Med Care. 2019;57:133 139. https://doi.org/10.1097 /MLR.0000000000001029.

17. Yoon H, Jang Y, Vaughan PW, Garcia M. Older adults' internet use for health information: digital divide by race/ ethnicity and socioeconomic status. J ApplGerontolOff J South GerontolSoc. 2020;39(1):105-10. https://doi. org/10.1177/0733464818770772.

18. Sarkar U, Karter AJ, Liu JY, et al. Social disparities in internet patient portal use in diabetes: evidence that the digital divide extends beyond access. J Am Med Inform Assoc. 2011;18(3):318-21. https://doi.org/10.1136 /jamia.2010.006015.

19. Kichloo A, Albosta M, Dettloff K, et al. Telemedicine, the current COVID-19 pandemic and the future: a narrative review and perspectives moving forward in the USA. Fam Med Community Health. 2020;8(3). https://doi.org/10.1136 /fmch-2020-000530

20. Nouri S, Khoong EC, Lyles CR, Karliner L. Addressing equity in telemedicine for chronic disease management during the Covid-19 pandemic. NEJM Catal. Published online May 4, 2020. https://catalyst.nejm.org/doi/abs/10.1056 /CAT.20.0123. Accessed 23 June 2020.

21. Wright A, Salazar A, Mirica M, Volk LA, Schiff GD. The invisible epidemic: neglected chronic disease management during COVID-19. J Gen Intern Med. 2020;35(9):2816-7. https://doi.org/10.1007/s11606-020-06025-4.

22. Schenker Y, Karter AJ, Schillinger D, et al. The impact of limited English proficiency and physician language concordance on reports of clinical interactions among patients with diabetes: the DISTANCE study. Patient EducCouns. 2010;81(2):222-8. https://doi.org/10.1016/j. pec.2010.02.005.

23. Rodriguez JA, Clark CR, Bates DW. Digital health equity as a necessity in the 21 st century cures act era. JAMA. 2020;323(23):2381-2. https://doi.org/10.1001 /jama.2020.7858.

Publisher's note Springer Nature remains neutral with regard to jurisdictional claims in published maps and institutional affiliations. 